\title{
A global climatology for equatorial plasma bubbles in the topside ionosphere
}

\author{
L. C. Gentile ${ }^{1}$, W. J. Burke ${ }^{2}$, and F. J. Rich ${ }^{2}$ \\ ${ }^{1}$ Institute for Scientific Research, Boston College, Chestnut Hill, MA, USA \\ ${ }^{2}$ Space Vehicles Directorate, Air Force Research Laboratory, Hanscom AFB, MA, USA
}

Received: 8 April 2006 - Revised: 18 November 2005 - Accepted: 21 December 2005 - Published: 7 March 2006

\begin{abstract}
We have developed a global climatology of equatorial plasma bubble (EPB) occurrence based on evening sector plasma density measurements from polar-orbiting Defense Meteorological Satellite Program (DMSP) spacecraft during 1989-2004. EPBs are irregular plasma density depletions in the post-sunset ionosphere that degrade communication and navigation signals. More than 14400 EPBs were identified in $\sim 134000$ DMSP orbits. DMSP observations basically agree with Tsunoda's (1985) hypothesis that EPB rates peak when the terminator is aligned with the Earth's magnetic field, but there are also unpredicted offsets in many longitude sectors. We present an updated climatology for the full database from 1989-2004 along with new plots for specific phases of the solar cycle: maximum 1989-1992 and 1999-2002, minimum 1994-1997, and transition years 1993, 1998, and 2003. As expected, there are significant differences between the climatologies for solar maximum and minimum and between the two solar maximum phases as well. We also compare DMSP F12, F14, F15, and F16 observations at slightly different local times during 2000-2004 to examine local time effects on EPB rates. The global climatologies developed using the DMSP EPB database provide an environmental context for the long-range prediction tools under development for the Communication/Navigation Outage Forecasting System (C/NOFS) mission.
\end{abstract}

Keywords. Ionosphere (Electric fields and currents; Equatorial ionosphere; Plasma waves and instabilities)

\section{Introduction}

In preparation for the Communication/Navigation Outage Forecasting System (C/NOFS) mission, we examined plasma density measurements from sensors on spacecraft of the Defense Meteorological Satellite Program (DMSP) and developed a global climatology of equatorial plasma bubble (EPB) occurrence to facilitate improvements in ionospheric models.

Correspondence to: L. C. Gentile

(louise.gentile@hanscom.af.mil)
EPBs are nonlinear evolutions of the generalized RayleighTaylor (R-T) instability in the post-sunset ionosphere in which bottomside plasma interchanges with plasma near and above the peak of the F-layer. Plasma irregularities with scale sizes of meters to kilometers form within these density depletions. As these large-scale irregularities propagate into the topside ionosphere, the smaller-scale irregularities within them diffract radio waves and cause severe degradation of communication and navigation signals at low magnetic latitudes. The C/NOFS mission objective is to learn to forecast the low-latitude ionospheric disturbances that cause these disruptions.

As a guide for our EPB studies we use a formula for the linear growth rate $\gamma$ of the generalized R-T instability derived by Zalesak and Ossakow (1982) and adapted by Sultan (1996):

$\gamma=\frac{\Sigma_{P}^{F}}{\Sigma_{P}^{E}+\Sigma_{P}^{F}}\left(V_{p}-U_{n}^{P}-\frac{g_{L}}{v_{\text {in }}^{e f f}}\right) \frac{1}{L_{n}}-R_{T}$,

where $\mathrm{g}_{\mathrm{L}}$ represents the downward acceleration due to gravity, $\mathrm{V}_{\mathrm{p}}=\boldsymbol{E} \times \boldsymbol{B} / \mathrm{B}^{2}$ the vertical component of plasma drift due to the zonal component of the electric field $\boldsymbol{E}$ at the magnetic equator, $\mathrm{U}_{\mathrm{n}}^{\mathrm{P}}$ the vertical component of neutral wind velocity perpendicular to $\boldsymbol{B}$, and $v_{\text {in }}^{\text {eff }}$ the effective, flux-tube integrated, F-region, ion-neutral collision frequency weighted by number density in the flux tube. $L_{n}$ is the scale length of the vertical gradient of the flux-tube integrated plasma density measured at the equator. $\mathrm{R}_{\mathrm{T}}$ is the flux-tube integrated recombination rate (Basu, 1997). $\Sigma_{P}^{E}$ and $\Sigma_{P}^{F}$ are the contributions to $\Sigma_{P}$ from the $\mathrm{E}$ and $\mathrm{F}$ regions, respectively. $\mathrm{L}_{\mathrm{n}}$ is positive in the bottomside of the F-layer in the post-sunset ionosphere. Eastward components of $\boldsymbol{E}$ and downward components of $\boldsymbol{U}_{\mathrm{n}}^{\mathrm{P}}$ contribute to a positive growth rate $\gamma$. Growth periods, $1 / \gamma$, are generally $\sim 10 \mathrm{~min}$, and large-amplitude irregularities develop over several growth periods. Since $g$ and $\boldsymbol{B}$ are constants at a given location, R-T growth rates are controlled by the variability of $\mathrm{E}_{0}, \mathrm{U}_{\mathrm{n}}^{\mathrm{P}}, \Sigma_{P}^{E}, \Sigma_{P}^{F}, v_{i n}^{e f f}, \mathrm{R}_{\mathrm{T}}$, and through the flux-tube integrated quantities by the height of the F-layer. 
There is a relatively small window of opportunity in the post sunset ionosphere for EPBs to develop. Shortly after dusk, as polarization charges increase in the vicinity of the terminator, the ionosphere rises and instabilities form. As the vertical drift slows and reverses, the formation of instabilities is inhibited. The seasonal-longitudinal model of Tsunoda (1985) predicted that EPB rates should peak near times when $\alpha$, the angle between the magnetic declination $\delta$ and the dusk terminator, is equal to zero, and the E-region conductance allows bubbles to grow most rapidly.

DMSP spacecraft fly in circular, sun-synchronous polar orbits at an altitude of $848 \mathrm{~km}$ and an inclination of $98.7^{\circ}$. The orbital trajectories of the spacecraft used this study F9, F10, F12, F14, F15, and F16 all cross the magnetic equator in the post-sunset local time (LT) sector (19:00-22:00 LT). With the exception of F9, the ascending nodes of the spacecraft are on the dusk side of the Earth. "Sun-synchronous" orbits are fixed with respect to the mean LT. Over the course of a year, the true solar position varies by a few minutes around the mean LT, and drag lowers the orbit by about a kilometer. The initial orbits of F10 and F12 were not quite sun-synchronous. In all cases, the LT changes at a rate of $\sim 30$ min or less per year on orbit.

Although it may seem unusual to employ polar-orbiting satellites to study equatorial phenomena, Burke et al. (2003, 2004a,b) and Huang et al. (2001, 2002) developed an initial global climatology of EPB occurrence that clearly demonstrated the utility of DMSP spacecraft as useful platforms for EPB observations. Huang et al. (2001) surveyed EPB activity during solar maximum years 1989 and 1991. Seasonal versus longitudinal distributions of EPBs were in general agreement with previously reported satellite and ground-based measurements (Aarons, 1993) as well as the stormtime model of Scherliess and Fejer (1997). Huang et al. (2001) also found that the number of EPBs increased during the early stages of geomagnetic storms, but was suppressed during recovery. Encouraged by this success, Huang et al. (2002) extended their study to include seasonal and longitudinal distributions of EPBs from multiple DMSP spacecraft over a full solar cycle from 1989-2000. Their results suggest that penetration electric fields are the driving force behind many stormtime EPBs. Burke et al. (2003) then compared DMSP EPB observations with coordinated ground measurements from the Jicamarca unattended long-term studies of the ionosphere and atmosphere (JULIA) radar and a scintillation monitor in Ancon, Peru, and determined that the seasonally averaged occurrence rates correlated closely despite the fact that many ionospheric disturbances that cause S4 fluctuations do not reach DMSP altitudes. Burke et al. (2003) also concluded that EPBs tend to occur in sporadic clusters rather than at regular intervals.

Burke et al. (2004a) then presented a global climatology of EPB rates for a full solar cycle 1989-2000 in a color contour plot similar to equatorial spread $\mathrm{F}$ occurrence patterns devised by Sultan (priv. comm., 2000). In general, the Burke et al. (2004a) climatology agreed with Tsunoda's (1985) model, but unpredicted offsets were evident in several longitude sectors, particularly in the Atlantic-Africa region. Burke et al. (2004b) updated the climatology for 1989-2002 and demonstrated that DMSP EPB observations were wellcorrelated with plasma density measurements from the Republic of China Satellite (ROCSAT-1) in a $35^{\circ}$ inclination orbit at $650 \mathrm{~km}$ when the satellites crossed the same longitude sector within $+/-15 \mathrm{~min}$. Furthermore, the occurrence rates of EPBs observed by ROCSAT-1 peaked around the LT of the orbital paths of the DMSP satellites, confirming that the spacecraft were well-placed to observe EPBs. Both DMSP and ROCSAT-1 observed the vast majority of the EPBs within $+/-20^{\circ}$ of the equator (Burke et al., 2004b).

Burke et al. (2004b) also examined scintillation measurements from a network of GPS receivers in South America. Based on unexpected minima in EPB rates near the west coast of South America and significant differences in scintillation measurements looking east and west from receiver sites, they proposed that particle precipitation from the inner radiation belt affects global distributions of EPBs and the climatology of radiowave scintillations. Enhanced drift loss cone precipitation of inner belt electrons to the west of the South Atlantic Anomaly (Luhmann and Vampola, 1977) would increase E-layer conductance and thus inhibit nonlinear EPB growth. This present study provides a more detailed picture of trends in EPB rates with new climatology plots for specific phases of the solar cycle and compares F12, F14, F15, and F16 results at various local times for 2000-2004. The long range objective is to facilitate improvements in ionospheric models that will enhance our ability to forecast and nowcast ionospheric disturbances during the C/NOFS era.

\section{Instrumentation}

DMSP spacecraft average 14 orbits of $\sim 104$ min each per day for a total of $\sim 5100$ orbits per year. Satellites twice traverse all magnetic latitudes between $\pm 20^{\circ}$ MLat within a narrow LT range during every orbit and regress $\sim 25^{\circ}$ in longitude between ascending nodes. The Special SensorIons, Electrons, and Scintillation (SSIES) measures plasma densities, temperatures, and drift motions. SSIES includes a spherical Langmuir probe mounted on an $0.8-\mathrm{m}$ boom to measure the densities and temperatures of ambient electrons and three ion sensors mounted on a conducting plate facing toward the ram direction (Rich and Hairston, 1994): (1) an ion trap to measure total ion density, (2) an ion drift meter to measure horizontal $\left(\mathrm{V}_{\mathrm{H}}\right)$ and vertical $\left(\mathrm{V}_{\mathrm{V}}\right)$ cross-track components of plasma drifts, and (3) a retarding potential analyzer to measure ion temperatures and in-track components of plasma drift $\mathrm{V}_{\|}$. For the DMSP studies, we examined evening sector SSIES plasma densities plotted as a function of magnetic latitude. This captures the existence of plasma depletions with scale sizes ranging from tens to hundreds of kilometers and ignores the existence of plasma irregularities with smaller scale sizes of meters to a few kilometers. 
Table 1. Summary of DMSP EPB observations by year, spacecraft, LT of spacecraft orbit, number of orbits, number of orbits with EPBs, and number of orbits with EPBs classified by the depth of the deepest depletion $\delta \mathrm{N}$ with respect to the nearby undisturbed plasma density: M-0 if $\delta N \leq 2 ; M-1$ if $2<\delta N \leq 10 ; M-2$ if $10<\delta N \leq 100 ;$ M-3 if $\delta N>100$ (c.f. Fig. 1 of Huang et al., 2001).

\begin{tabular}{|c|c|c|c|c|c|c|c|c|}
\hline Year & Satellite & LT (Hr) & Orbits & EPBs & M-0 & M-1 & M-2 & M-3 \\
\hline 1989 & F09 & 21 & 5121 & 1109 & 297 & 762 & 50 & $\mathbf{0}$ \\
\hline 1990 & F09 & 20.9 & 5091 & 1089 & 289 & 735 & 62 & 3 \\
\hline 1991 & F09 & 20.7 & 5040 & 1024 & 304 & 664 & 55 & 1 \\
\hline 1991 & F10 & 20 & 4925 & 675 & 218 & 351 & 86 & 20 \\
\hline 1992 & F10 & 20.7 & 5043 & 755 & 306 & 406 & 41 & 2 \\
\hline 1993 & F10 & 21.3 & 5092 & 389 & 186 & 191 & 11 & 1 \\
\hline 1994 & F10 & 21.7 & 3749 & 121 & 53 & 58 & 10 & 0 \\
\hline 1994 & F12 & 21.4 & 1575 & 42 & 33 & 8 & 1 & 0 \\
\hline 1995 & F12 & 21.4 & 4976 & 130 & 51 & 71 & 8 & $\mathbf{0}$ \\
\hline 1996 & F12 & 21.5 & 5122 & 73 & 35 & 36 & 2 & 0 \\
\hline 1997 & F12 & 21.4 & 3343 & 47 & 24 & 23 & $\mathbf{0}$ & 0 \\
\hline 1997 & F14 & 20.6 & 3104 & 51 & 18 & 32 & 1 & $\mathbf{0}$ \\
\hline 1998 & F12 & 21.2 & 4123 & 283 & 106 & 169 & 7 & 1 \\
\hline 1998 & F14 & 20.7 & 4993 & 275 & 129 & 138 & 8 & 0 \\
\hline 1999 & F12 & 20.8 & 4290 & 431 & 83 & 330 & 13 & 5 \\
\hline 1999 & F14 & 20.8 & 4839 & 460 & 167 & 285 & 6 & 2 \\
\hline 2000 & F12 & 20.3 & 4556 & 637 & 85 & 509 & 41 & 2 \\
\hline 2000 & F14 & 20.7 & 4969 & 821 & 298 & 493 & 26 & 4 \\
\hline 2000 & F15 & 21.3 & 5060 & 1034 & 377 & 620 & 30 & 7 \\
\hline 2001 & F12 & 19.8 & 4557 & 404 & 60 & 305 & 34 & 5 \\
\hline 2001 & F14 & 20.6 & 4787 & 861 & 174 & 643 & 38 & 6 \\
\hline 2001 & F15 & 21.5 & 5095 & 1014 & 292 & 699 & 20 & 3 \\
\hline 2002 & F14 & 20.3 & 4889 & 781 & 160 & 563 & 55 & 3 \\
\hline 2002 & F15 & 21.5 & 5113 & 991 & 279 & 672 & 40 & 0 \\
\hline 2003 & F14 & 19.9 & 4732 & 156 & 37 & 108 & 5 & 6 \\
\hline 2003 & F15 & 21.4 & 5149 & 385 & 84 & 275 & 15 & 11 \\
\hline 2004 & F14 & 19.6 & 4777 & 30 & 10 & 14 & 6 & 0 \\
\hline 2004 & F15 & 21.3 & 5123 & 267 & 63 & 180 & 14 & 10 \\
\hline 2004 & F16 & 19.9 & 5131 & 77 & 18 & 51 & 7 & 1 \\
\hline Totals & & & 134364 & 14412 & 4236 & 9391 & 692 & 93 \\
\hline
\end{tabular}

\section{Observations}

This study extends the EPB climatology of Burke et al. (2004a, b) with additional data from F12 for 1999-2000, F14 and F15 for 2003, and F14, F15, and F16 for 2004. Table 1 summarizes the DMSP EPB observations by year, spacecraft, LT of the spacecraft orbit, number of orbits, number of orbits with EPBs, and number of orbits with EPBs classified by the depth of the deepest depletion $\delta \mathrm{N}$ with respect to the nearby undisturbed plasma density: $\mathrm{M}-0$ if $\delta \mathrm{N} \leq 2 ; \mathrm{M}-1$ if $2<\delta \mathrm{N} \leq 10 ; \mathrm{M}-2$ if $10<\delta \mathrm{N} \leq 100 ; \mathrm{M}-3$ if $\delta \mathrm{N}>100$ (c.f. Fig. 1 of Huang et al. (2001)). Plasma irregularities within the depletions made it difficult to count individual EPBs; each cluster was classified as one EPB according to the depth of the deepest depletion during that equatorial pass. Data were sorted by the longitude of the satellite's equatorial crossing rather than the specific longitude of individual EPBs.
DMSP data reveal solar cycle, seasonal, and longitudinal effects. The number of orbits in which an individual satellite encountered EPBs ranged from $\sim 1100$ per year during solar maximum to $<100$ at solar minimum. In general, more EPBs were observed around the equinoxes in March-April and September-October than near the solstices in June-July and December-January (Huang et al., 2002). EPB rates were lower in the Pacific where the equatorial magnetic field is $>3 * 10^{4} \mathrm{nT}$ than in the American-Atlantic sector where it is $<3^{*} 10^{4}$ nT (Fig. 10 of Burke et al., 2004b). During solar minimum, $\sim 1 / 3$ of the EPBs were observed during times when plots of Dst had significant and sustained negative slopes suggesting the presence of penetration electric fields (Huang et al., 2002). EPBs of all types occur in all phases of the solar cycle, but there were significantly more EPBs, especially the deeper M-2 and M-3 EPBs, during solar maximum. Dst plots indicate that most M-3 EPBs occur during the main phase of magnetic storms (Huang et al., 2001). 
Table 2. Number of DMSP orbits per month in each longitude sector for the full DMSP EPB database 1989-2004.

\begin{tabular}{ccccccccccccc}
\hline Longitude & Jan & Feb & Mar & Apr & May & Jun & Jul & Aug & Sept & Oct & Nov & Dec \\
\hline $\mathbf{- 1 8 0}$ & 364 & 339 & 359 & 351 & 385 & 368 & 409 & 391 & 372 & 381 & 348 & 378 \\
$\mathbf{- 1 6 5}$ & 394 & 392 & 398 & 404 & 433 & 421 & 428 & 418 & 435 & 428 & 366 & 447 \\
$\mathbf{- 1 5 0}$ & 441 & 396 & 443 & 424 & 451 & 454 & 469 & 457 & 464 & 462 & 430 & 450 \\
$\mathbf{- 1 3 5}$ & 463 & 442 & 473 & 468 & 485 & 468 & 477 & 473 & 484 & 491 & 453 & 474 \\
$\mathbf{- 1 2 0}$ & 472 & 414 & 461 & 437 & 473 & 459 & 487 & 477 & 483 & 475 & 430 & 479 \\
$\mathbf{- 1 0 5}$ & 450 & 424 & 464 & 452 & 475 & 456 & 478 & 468 & 466 & 467 & 449 & 455 \\
$\mathbf{- 9 0}$ & 451 & 422 & 450 & 453 & 481 & 472 & 481 & 476 & 482 & 475 & 437 & 470 \\
$\mathbf{- 7 5}$ & 486 & 434 & 495 & 469 & 505 & 492 & 490 & 504 & 487 & 486 & 469 & 468 \\
$\mathbf{- 6 0}$ & 499 & 481 & 505 & 501 & 528 & 517 & 522 & 522 & 537 & 538 & 483 & 518 \\
$\mathbf{- 4 5}$ & 531 & 481 & 516 & 508 & 531 & 524 & 527 & 548 & 529 & 537 & 504 & 525 \\
$\mathbf{- 3 0}$ & 495 & 452 & 496 & 480 & 504 & 487 & 499 & 509 & 520 & 507 & 464 & 489 \\
$\mathbf{- 1 5}$ & 457 & 430 & 454 & 470 & 481 & 461 & 482 & 471 & 489 & 488 & 453 & 470 \\
$\mathbf{0}$ & 477 & 430 & 481 & 461 & 482 & 470 & 490 & 486 & 489 & 503 & 466 & 496 \\
$\mathbf{1 5}$ & 447 & 415 & 454 & 446 & 468 & 450 & 456 & 459 & 473 & 478 & 415 & 458 \\
$\mathbf{3 0}$ & 461 & 431 & 465 & 456 & 470 & 464 & 480 & 463 & 469 & 463 & 433 & 475 \\
$\mathbf{4 5}$ & 468 & 427 & 471 & 452 & 483 & 459 & 485 & 490 & 481 & 481 & 442 & 471 \\
$\mathbf{6 0}$ & 467 & 442 & 479 & 476 & 487 & 477 & 493 & 489 & 473 & 486 & 457 & 475 \\
$\mathbf{7 5}$ & 457 & 427 & 484 & 467 & 482 & 463 & 496 & 487 & 487 & 476 & 455 & 483 \\
$\mathbf{9 0}$ & 464 & 438 & 463 & 452 & 484 & 471 & 484 & 495 & 487 & 489 & 443 & 480 \\
$\mathbf{1 0 5}$ & 484 & 431 & 480 & 459 & 473 & 472 & 475 & 482 & 487 & 506 & 455 & 455 \\
$\mathbf{1 2 0}$ & 461 & 445 & 478 & 473 & 489 & 466 & 473 & 488 & 490 & 490 & 450 & 483 \\
$\mathbf{1 3 5}$ & 477 & 428 & 472 & 461 & 480 & 474 & 485 & 486 & 484 & 499 & 451 & 483 \\
$\mathbf{1 5 0}$ & 458 & 428 & 470 & 462 & 464 & 456 & 479 & 469 & 469 & 479 & 443 & 457 \\
$\mathbf{1 6 5}$ & 485 & 451 & 487 & 472 & 490 & 487 & 491 & 496 & 495 & 503 & 460 & 476 \\
$\mathbf{1 8 0}$ & 364 & 339 & 359 & 351 & 385 & 368 & 409 & 391 & 372 & 381 & 348 & 378 \\
\hline
\end{tabular}

Table 3. Number of orbits with EPBs per month in each longitude sector for the full DMSP EPB database 1989-2004.

\begin{tabular}{ccccccccccccc}
\hline Longitude & Jan & Feb & Mar & Apr & May & Jun & Jul & Aug & Sept & Oct & Nov & Dec \\
\hline $\mathbf{- 1 8 0}$ & 4 & 8 & 20 & 46 & 42 & 36 & 64 & 55 & 63 & 20 & 8 & 1 \\
$\mathbf{- 1 6 5}$ & 0 & 8 & 16 & 31 & 29 & 30 & 44 & 76 & 62 & 28 & 10 & 4 \\
$\mathbf{- 1 5 0}$ & 6 & 11 & 32 & 34 & 28 & 24 & 23 & 54 & 77 & 34 & 10 & 1 \\
$\mathbf{- 1 3 5}$ & 10 & 12 & 44 & 43 & 31 & 24 & 18 & 51 & 65 & 33 & 13 & 7 \\
$\mathbf{- 1 2 0}$ & 6 & 35 & 87 & 67 & 41 & 14 & 17 & 50 & 96 & 63 & 27 & 11 \\
$\mathbf{- 1 0 5}$ & 27 & 44 & 109 & 91 & 21 & 14 & 11 & 39 & 112 & 91 & 28 & 22 \\
$\mathbf{- 9 0}$ & 37 & 60 & 121 & 59 & 10 & 7 & 7 & 29 & 103 & 90 & 49 & 30 \\
$\mathbf{- 7 5}$ & 106 & 95 & 89 & 14 & 3 & 2 & 5 & 10 & 62 & 91 & 99 & 87 \\
$\mathbf{- 6 0}$ & 171 & 120 & 108 & 14 & 1 & 0 & 7 & 10 & 60 & 129 & 158 & 183 \\
$\mathbf{- 4 5}$ & 136 & 107 & 112 & 28 & 1 & 2 & 2 & 3 & 64 & 178 & 191 & 181 \\
$\mathbf{- 3 0}$ & 101 & 111 & 146 & 50 & 7 & 5 & 3 & 11 & 97 & 187 & 152 & 114 \\
$\mathbf{- 1 5}$ & 67 & 111 & 172 & 120 & 41 & 9 & 24 & 54 & 146 & 165 & 131 & 77 \\
$\mathbf{0}$ & 35 & 68 & 151 & 160 & 96 & 28 & 56 & 113 & 174 & 169 & 85 & 35 \\
$\mathbf{1 5}$ & 19 & 36 & 146 & 161 & 90 & 47 & 61 & 82 & 135 & 128 & 56 & 19 \\
$\mathbf{3 0}$ & 18 & 37 & 92 & 126 & 54 & 16 & 24 & 54 & 88 & 85 & 43 & 15 \\
$\mathbf{4 5}$ & 33 & 54 & 95 & 89 & 20 & 5 & 6 & 19 & 64 & 75 & 53 & 18 \\
$\mathbf{6 0}$ & 40 & 73 & 75 & 57 & 8 & 2 & 4 & 16 & 50 & 67 & 46 & 21 \\
$\mathbf{7 5}$ & 28 & 67 & 89 & 54 & 15 & 6 & 0 & 16 & 56 & 69 & 18 & 8 \\
$\mathbf{9 0}$ & 24 & 51 & 87 & 53 & 19 & 2 & 6 & 24 & 62 & 51 & 13 & 1 \\
$\mathbf{1 0 5}$ & 11 & 34 & 76 & 54 & 23 & 3 & 8 & 27 & 63 & 59 & 8 & 3 \\
$\mathbf{1 2 0}$ & 8 & 27 & 81 & 48 & 27 & 6 & 16 & 35 & 66 & 45 & 11 & 3 \\
$\mathbf{1 3 5}$ & 11 & 20 & 57 & 51 & 30 & 7 & 19 & 34 & 62 & 36 & 5 & 1 \\
$\mathbf{1 5 0}$ & 6 & 5 & 58 & 65 & 37 & 30 & 41 & 50 & 53 & 26 & 6 & 3 \\
$\mathbf{1 6 5}$ & 4 & 9 & 60 & 86 & 60 & 45 & 73 & 70 & 61 & 21 & 9 & 3 \\
$\mathbf{1 8 0}$ & 4 & 8 & 20 & 46 & 42 & 36 & 64 & 55 & 63 & 20 & 8 & 1 \\
\hline
\end{tabular}

Note from Table 1 that the number of DMSP spacecraft with available data varied from year to year. For example, with F9 and F10 both operational in 1991, we actually have five "satellite years" of data for 1989-1992. For 1994-1997, there are six "satellite years" of data, and for 1999-2002 there are ten. In the transition years, we have data from either one $(1993)$ or two $(1998,2003)$ satellites. EPB rates were calculated as the ratio of the number of orbits in which 

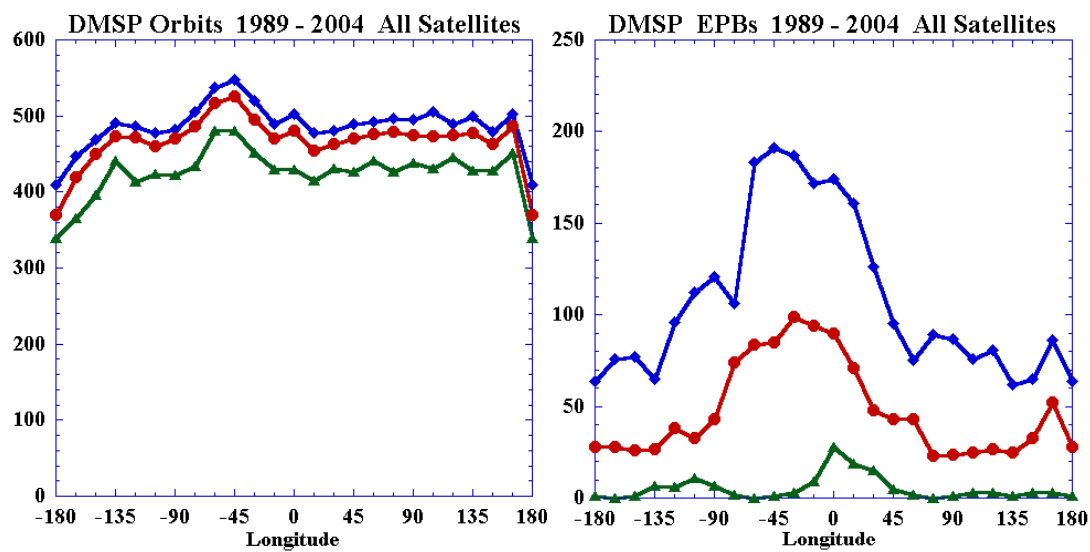

Fig. 1. Maximum, minimum, and median number of orbits (left) and EPBs (right) per month in each longitude sector for 1989-2004 based on data in Tables 2 and 3. Overall, the number of orbits per longitude/month bin ranged from 339 to 548. Highest EPB rates occurred in the America-Atlantic-Africa sector.
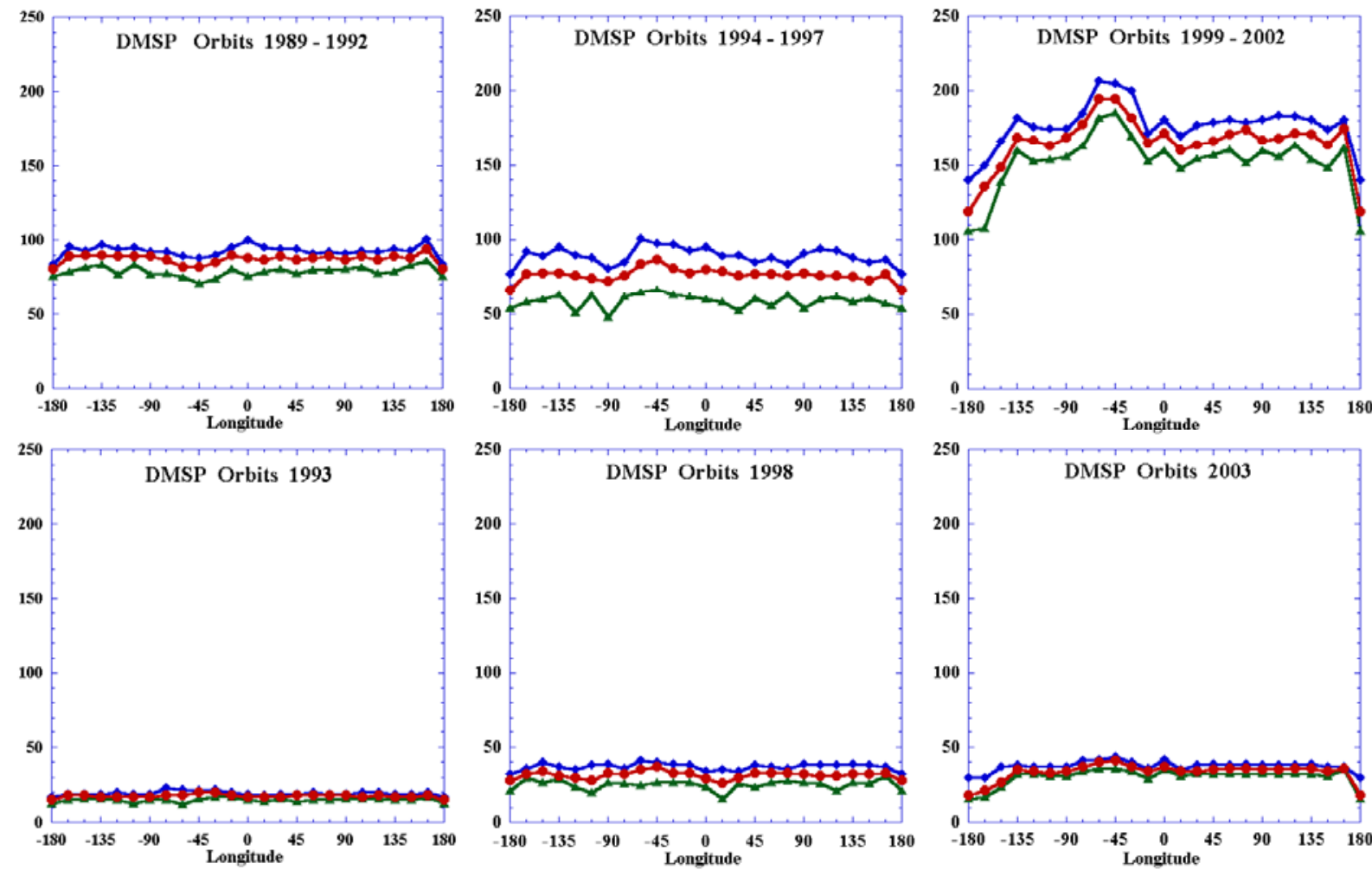

Fig. 2. Plots of the maximum, minimum, and median number of orbits per month sampled in each $15^{\circ}$ longitude sector for each phase of the solar cycle: maximum 1989-1992, minimum 1994-1997, maximum 1999-2002, and transition years 1993, 1998, and 2003. Data are derived from Tables 2 and 3 for the appropriate years.

at least one EPB was detected divided by the total number of orbits for each month of the year in 24 longitude sectors of $15^{\circ}$. There were on average $\sim 18$ orbits per month for each satellite in each longitude bin. To provide some perspective on the size of the statistical samples used to calculate the EPB rates, Table 2 is an array of the total number of orbits per month and Table 3 the number of orbits with EPBs in each $15^{\circ}$ longitude sector for all the spacecraft listed in Table 1.
Figure 1 plots the maximum, minimum, and median number of orbits (left) and EPBs (right) per month in each longitude sector for 1989-2004. The number of orbits per longitude/month bin ranged from 339 to 548 . The maximum number of EPBs (191) occurred between $-60^{\circ}$ and $-45^{\circ}$, while overall the highest rates were observed in the AmericaAtlantic-Africa sector. Figure 2 includes similar orbit plots for each phase of the solar cycle. 


\section{DMSP EPB Rates 1989 - 2004}

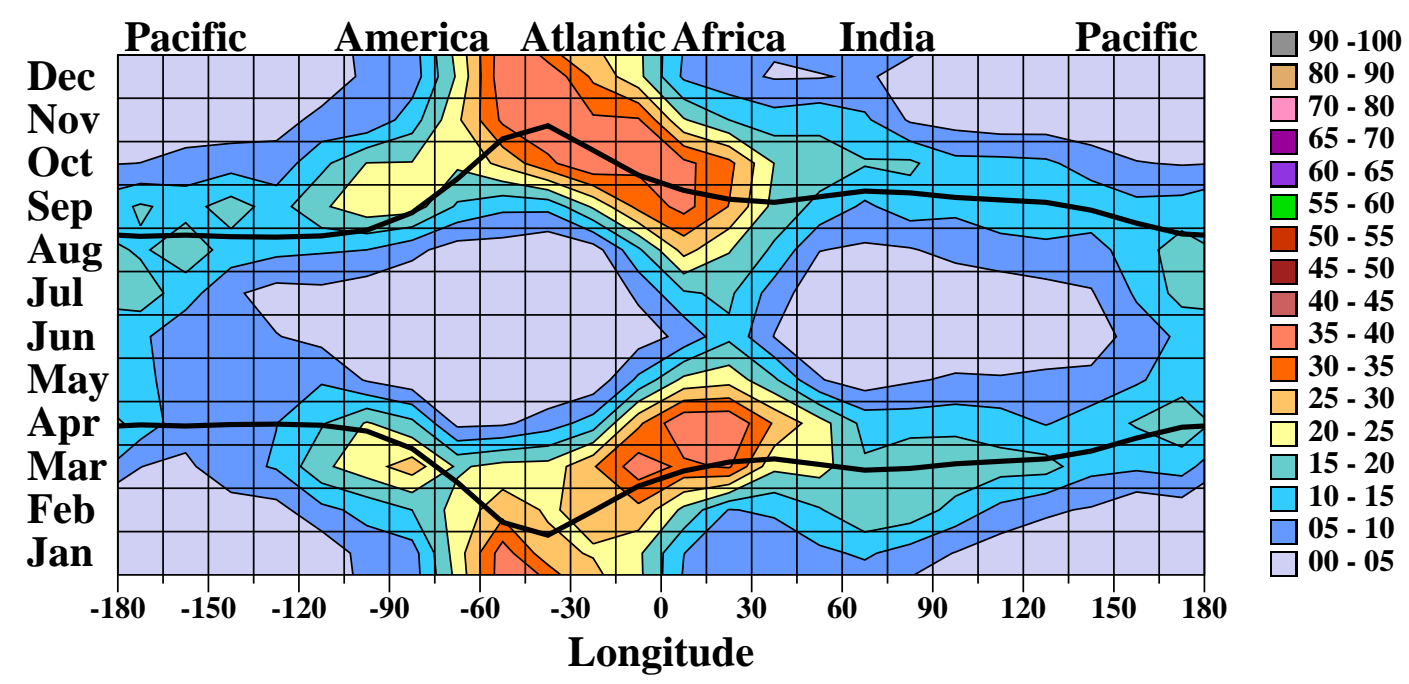

Fig. 3. Contour plot representing EPB rates for 1989-2004 on a month versus longitude grid. Colors represent the rates as indicated. Black lines mark the two times per year when $\alpha=0^{\circ}$ within each longitude bin.

DMSP EPB Rates 1989 - 1992

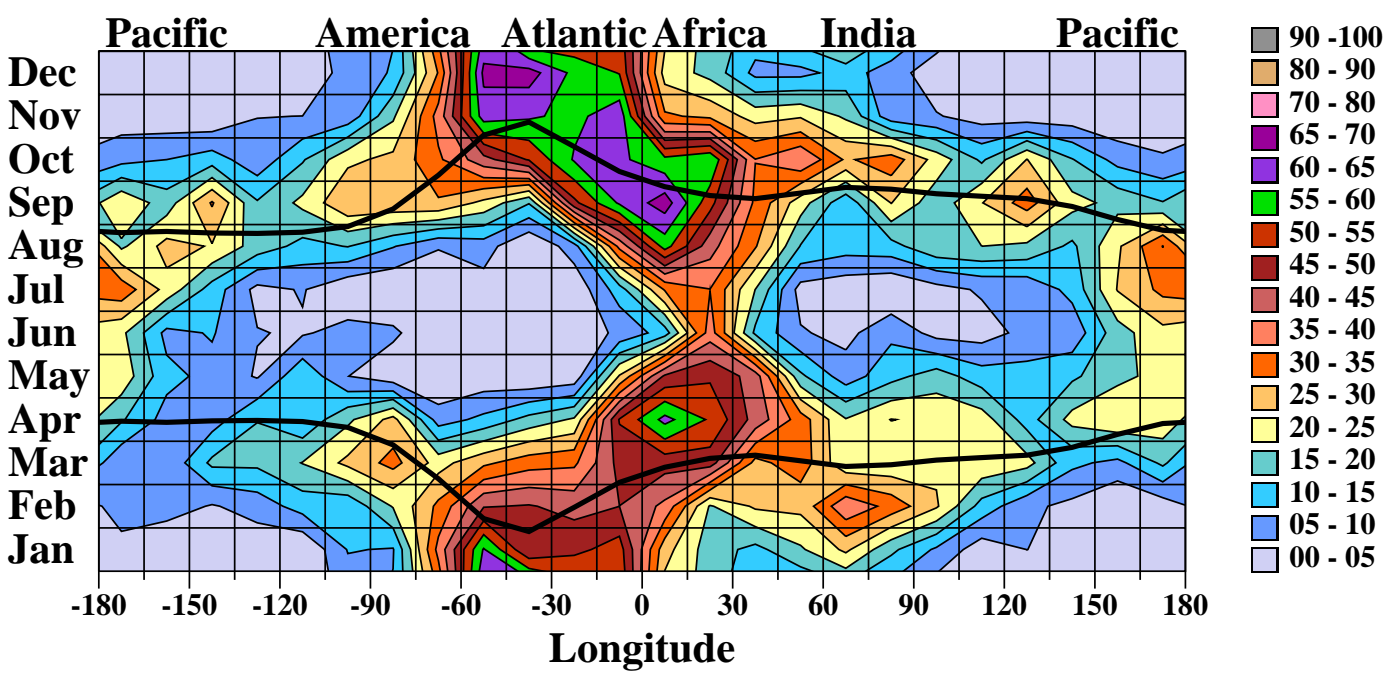

Fig. 4. Contour plot of EPB rates for solar maximum 1989-1992 in the same format as Fig. 3. EPBs occurred throughout the year in the Atlantic-Africa sector; rates were $>60 \%$ in January, April, and September to December.

Figure 3 is a rate of occurrence map for EPBs observed by DMSP satellites from 1989-2004 plotted on a month-versuslongitude grid. Longitude ranges from $-180^{\circ}$ to $180^{\circ}$ in 24 bins of $15^{\circ}$. Colors represent rates as indicated. Superimposed black lines mark the days when $\alpha=0^{\circ}$.

In general, the DMSP observations agree with Tsunoda's hypothesis (1985); most EPBs were observed near days when $\alpha=0^{\circ}$. However, the maximum rates do not always coincide with these days and the significant offsets apparent in many longitude sectors, particularly in the AtlanticAfrica region, were not predicted by the model. Both seasonal and longitudinal effects are evident, with the highest
EPB rates (35\%-40\%) observed around the spring (MarchApril) and fall (September-October) equinoxes and the winter (December-January) solstice in the America-AtlanticAfrica region. Lowest rates $(0 \%-5 \%)$ occurred during January-February and November-December in the Pacific sector and June-July in the America-Atlantic and India sectors.

We then plotted EPB rates for solar maximum (19891992 and 1999-2002), minimum (1994-1997), and transition years $(1993,1998$, and 2003) based on the F10.7 index of solar radiation flux. In all phases of the solar cycle DMSP satellites observed more EPBs in the Atlantic-Africa 


\section{DMSP EPB Rates 1999 - 2002}

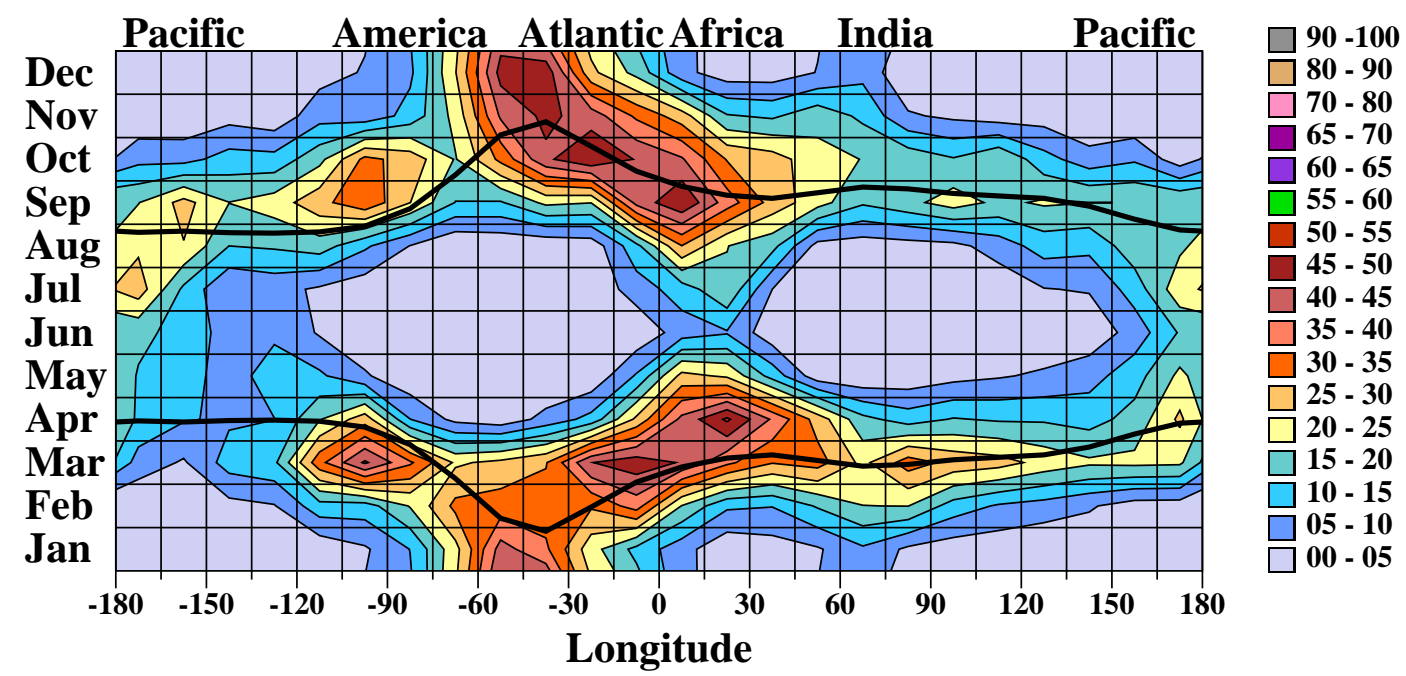

Fig. 5. Contour plot of EPB rates for solar maximum 1999-2002 in the same format as Fig. 3. Plot is fairly symmetric with similar maxima $(40 \%-50 \%)$ in the America-Atlantic-Africa sector both early and late in the year.

\section{DMSP EPB Rates 1994 - 1997}

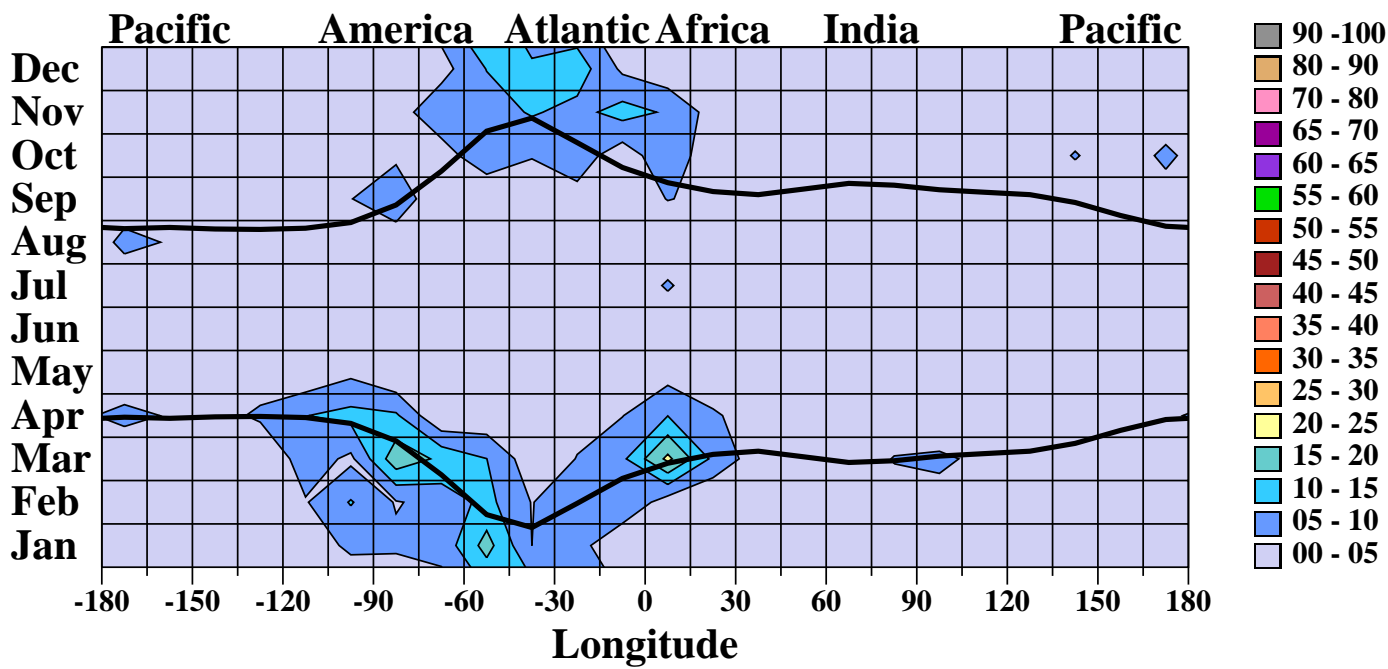

Fig. 6. Contour plot of EPB rates for solar minimum 1994-1997 in the same format as Fig. 3. EPB rates were generally $<=5 \%$. Highest rate $(21 \%)$ occurred in the Africa sector in March.

sector than in the Pacific. Figure 4 is the EPB climatology for 1989-1992, the maximum phase of Solar Cycle 23, plotted on a month-versus-longitude grid in a format similar to that of Fig. 3. EPBs were observed in significant numbers throughout the year in the Atlantic-Africa sector with the highest rates (60\%-70\%) occurring in January, April, and September-December. Also note that the maximum EPB rates were significantly higher than those during the maximum phase of Solar Cycle 24 (45\%-50\%) from 1999-2002 shown in Fig. 5. This is consistent with Huang et al. (2002) who reported a strong linear relationship between EPB rates and the yearly averaged F10.7 index both globally and in individual longitude sectors (c.f. Fig. 4 and Table 2 of Huang et al., 2002). Another notable difference is the remarkably symmetric distribution for 1999-2002: EPB rates were high $(40 \%-50 \%)$ in the Atlantic-Africa sector from January to April and September to December with distinct minima $(0 \%-5 \%)$ around the summer solstice in the America and India sectors.

The climatology plot for solar minimum 1994-1997 (Fig. 6) presents a striking contrast to the ones for solar maximum. EPB rates were generally 0\%-5\% except in the America-Atlantic-Africa sector and a few other isolated spots. Most EPBs were observed early in the year. Although 


\section{DMSP EPB Rates 1993}

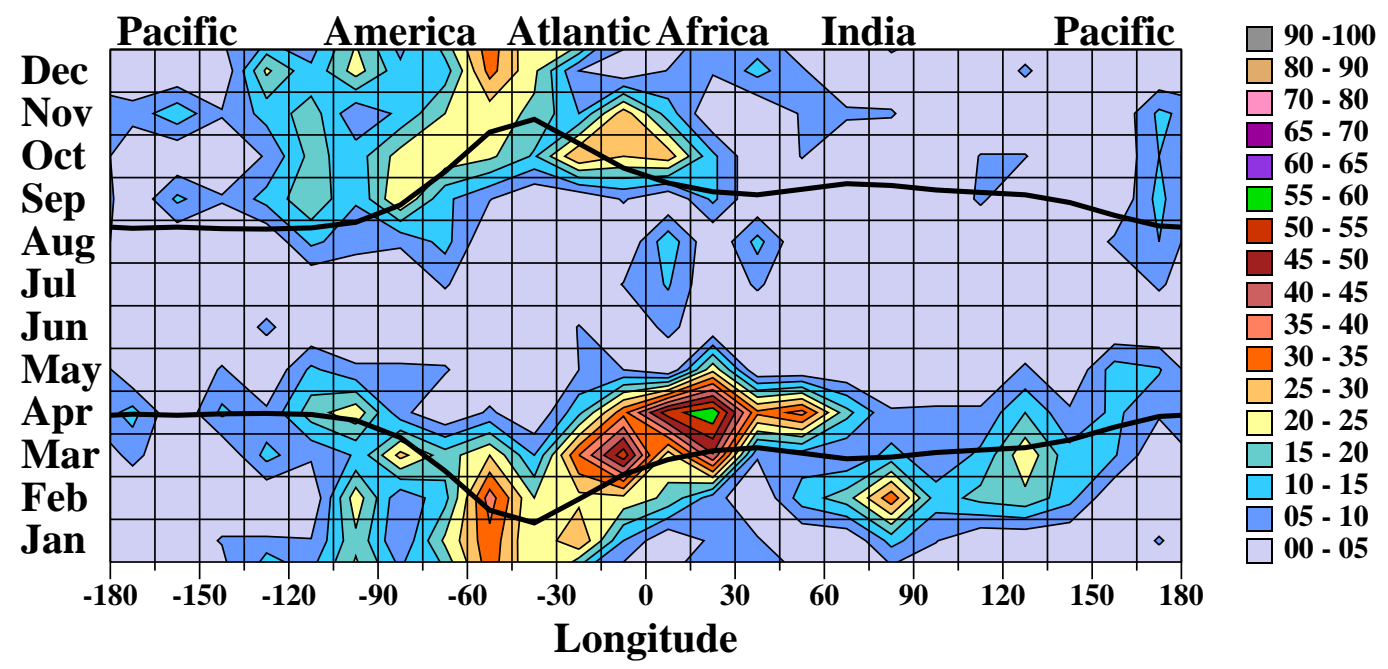

Fig. 7. EPB rates for transition year 1993 were highest (50\%-60\%) in the Atlantic-Africa sector during March-April.

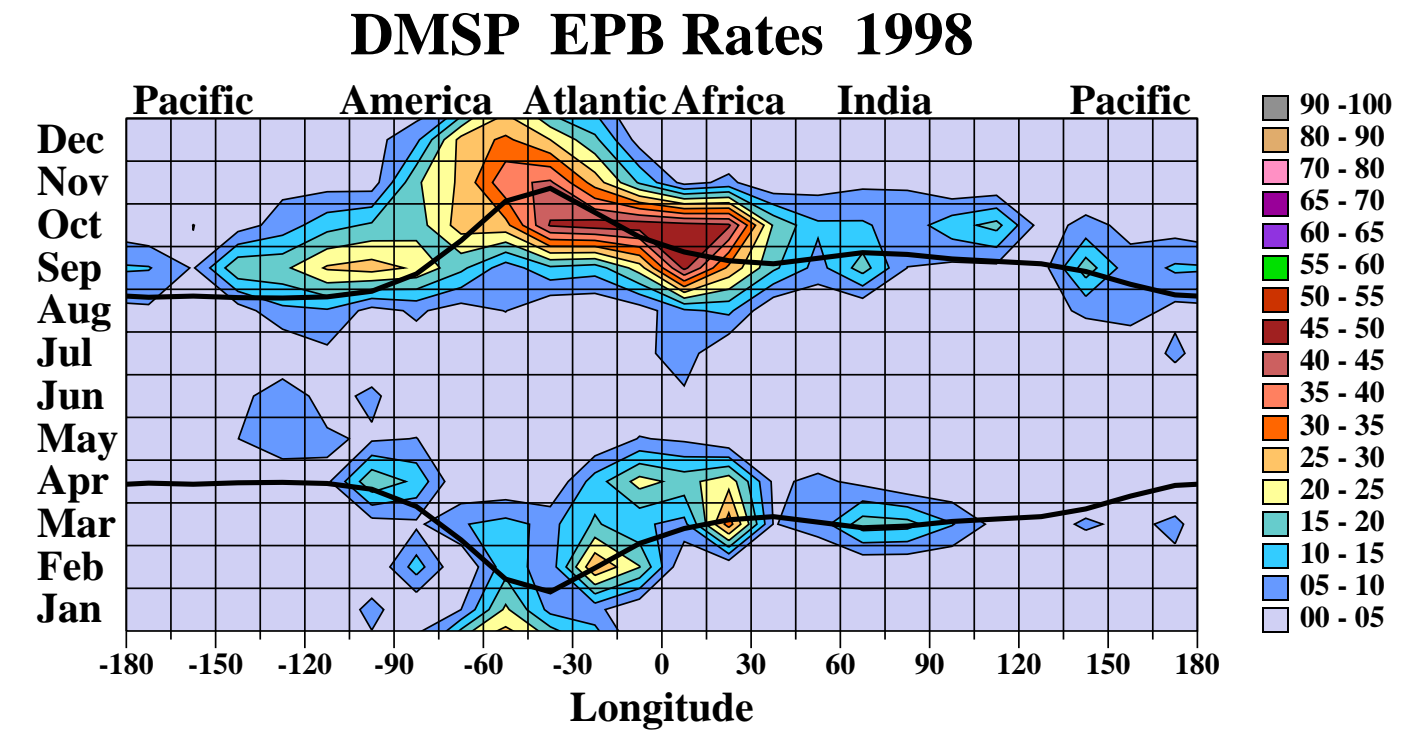

Fig. 8. For transition year 1998, highest EPB rates (45\%-50\%) occurred in the Atlantic-Africa sector late in the year.

the height distribution of EPBs remains an open question, ionospheric conditions during solar minimum may limit the number of EPBs that reach DMSP altitudes at the appropriate local times. The $\mathrm{C} / \mathrm{NOFS}$ satellite's $13^{\circ}$ inclination orbit with an apogee of $710 \mathrm{~km}$ and perigee of $375 \mathrm{~km}$ should maximize the probability of observing EPBs even under solar minimum conditions.

Figure 7 presents data for the 1993 transition year as the solar cycle declined. This is our smallest statistical sample with data from F10 only, but the plot shows similar trends in activity. EPB rates were higher early in the year with a maximum of 50\%-60\% during March-April in the AtlanticAfrica sector. A significant number of EPBs were encountered in the India sector in February, but by fall very few EPBs were observed in this area.
The trend reverses in 1998 (Fig. 8) with only a few localized hot spots in the Atlantic-Africa sector early in the year. EPB rates were significantly higher $(45 \%-50 \%)$ late in the year, September-October, in the Atlantic-Africa sector as the solar cycle approached maximum.

For 2003 (Fig. 9), the maximum rate (32\%) was lower than in 1993 or 1998, which seems reasonable for the declining phase of Solar Cycle 24. However, contrary to expectations, more EPBs were encountered late in the year in the AtlanticAfrica sector primarily because of the number of EPBs observed during the October-November solar storms.

In most of the climatology plots, observations from different local times were combined. Burke et al. (2004b) showed that ROCSAT-1 EPB rates for March-April 2000 and 2002 at $650-\mathrm{km}$ altitude varied with magnetic local time (MLT) 


\section{DMSP EPB Rates 2003}

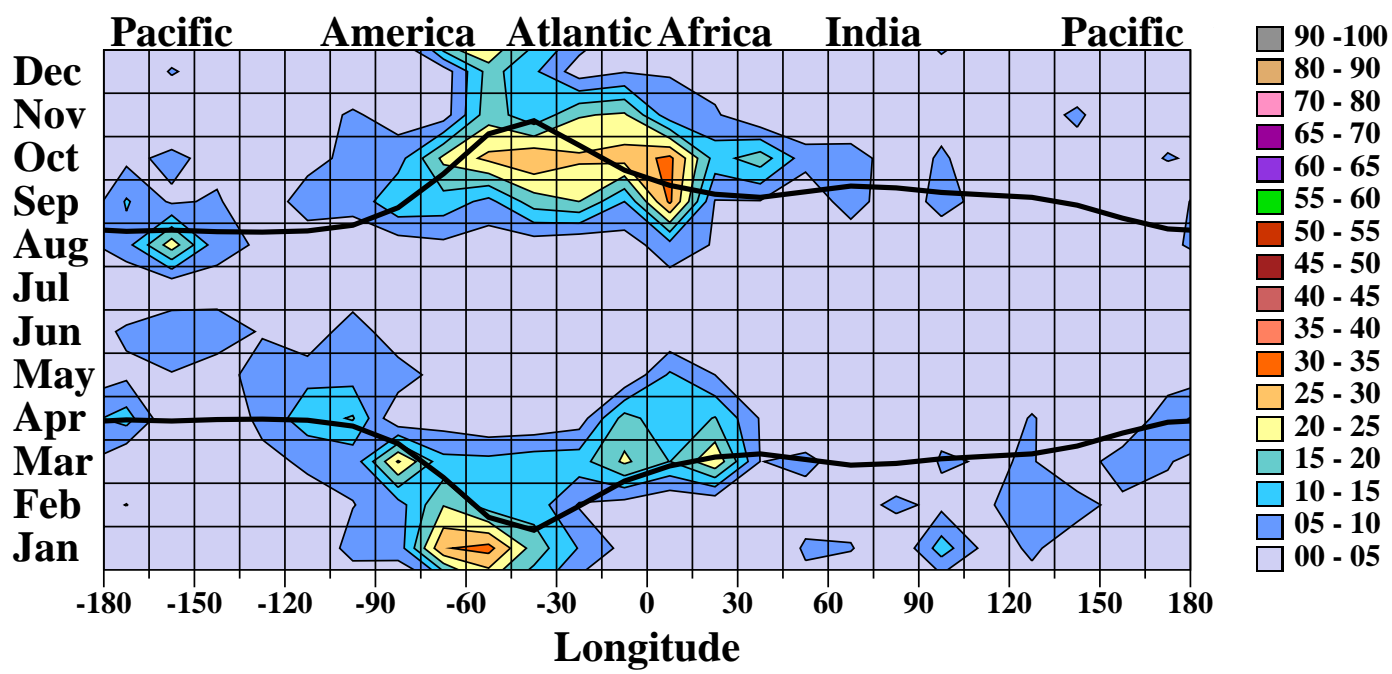

Fig. 9. EPB rates for transition year 2003 were higher in the Atlantic-Africa sector late in the year because of the number of EPBs during the October-November solar storms.

(cf. Fig. 8 of Burke et al., 2004b) from approximately zero near 18.7 MLT to a fairly consistent level from 20.3 MLT to midnight, the LT range of most of the DMSP orbits. For DMSP spacecraft, the average LT varies from year to year (see Table 1). To determine the variation in EPB rate as a function of LT for DMSP at $848 \mathrm{~km}$ we compared data from F12, F14, F15, and F16 for 2000 to 2004. This interval had two distinct advantages: several spacecraft were available simultaneously, and the maximum phase of the solar cycle yields the best statistics for EPBs. Of the four satellites, F15 was at the latest LT. Thus, we assumed that F15 was in the LT range when the occurrence rate was relatively constant ( 20.0-24.0 MLT). EPB rates for F15 from 2000 to 2004 were $20 \%, 20 \%, 19 \%, 7 \%$ and $5 \%$, respectively. To normalize the EPB rates for F15 and the other spacecraft in this time interval, we multiplied the occurrence rate for all spacecraft in a given year by a factor which normalized the F15 rate for that year at $20 \%$. This removed the influence of the solar cycle variation. Figure 10 shows that the normalized DMSP EPB rates rose from approximately zero near 19.3 MLT to a maximum near 21.0 MLT with the half-maximum rate at $\sim 20.0$ MLT. For ROCSAT-1, the half-maximum rate occurred at $\sim 19.5$ MLT. Assuming that this difference is due to the average speed of the rising bubbles, we estimate an upward drift speed of $\sim 110 \mathrm{~m} / \mathrm{s}$, which is within the expected range for EPBs.

\section{Discussion and Conclusions}

The DMSP EPB database includes 29 "satellite years" of evening sector observations from six polar-orbiting spacecraft during 1989-2004. This interval encompasses two solar maxima and a solar minimum, providing an opportunity to examine global distributions of EPBs for a variety of time

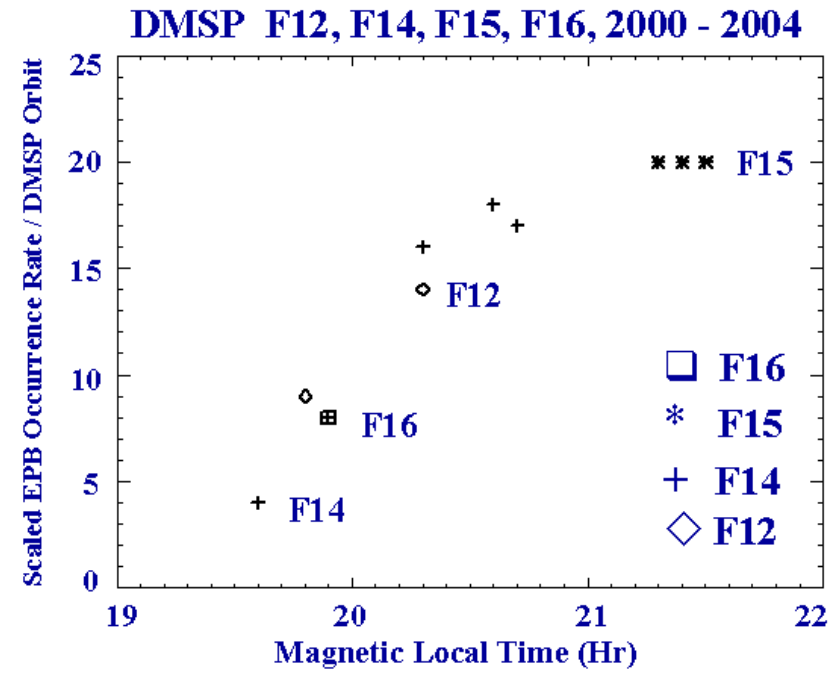

Fig. 10. LT distribution of EPBs observed from DMSP F12, F14, $\mathrm{F} 15$, and F16 at $848-\mathrm{km}$ altitude for 2000 to 2004 as a function of LT. Non-solar maximum years 2002-2004 were scaled to solar maximum years 2000-2001.

scales and solar cycle phases. The new climatology plots, tailored for specific years, provide a more detailed picture of trends in EPB occurrence rates at DMSP altitudes for a range of solar activity conditions. Seasonal and longitudinal distributions are consistent with those reported earlier by Burke et al. (2004a, b) with the highest occurrence rates in the America-Atlantic-Africa in all phases of the solar cycle. DMSP EPB rates correlate well with the F10.7 index of solar activity; significantly more EPBs were encountered during solar maximum years than during solar minimum conditions. This is consistent with two other manifestations of solar max- 
imum activity, the impact of increased EUV flux on ionospheric dynamics and the presence of penetration electric fields in the magnetosphere. It is important to keep in mind that the EPBs detected at DMSP altitudes are the result of nonlinear evolutions of the Rayleigh-Taylor instability that have reached the peak of the F-layer. Not all EPBs reach this altitude, especially during solar minimum. Burke et al. (2004b) found good agreement with ROCSAT-1 plasma density measurements in a low inclination orbit at $650 \mathrm{~km}$. However, the height distribution of EPBs remains an open question, a challenge which the C/NOFS mission is designed to address.

The primary objective of the DMSP studies has been to exploit global and continuous observations of plasma densities at low magnetic latitudes in the evening LT sector in preparation for the C/NOFS mission. As with any statistical study our methodology offers advantages and disadvantages relative to other techniques. Each DMSP spacecraft measures plasma densities at $848 \mathrm{~km}$ near a specific LT with equatorial crossings separated in longitude by $\sim 25^{\circ}$ from one orbit to the next. With this coarse filter a DMSP satellite misses many plasma bubbles. However, it does observe many internal irregularities within depletions as it follows the magnetic flux tube. While satellites such as Atmosphere Explorer E and ROCSAT-1 flying in low inclination orbits sample plasma density irregularities at all longitudes and local times, their operational lifetimes were limited to a few years. Burke et al. (2004b) demonstrated that if ROCSAT- 1 crossed an EPB at a given longitude and a DMSP satellite crossed the magnetic equator at the same longitude within \pm 15 min the probability of detecting the same depletion was nearly $100 \%$. In other words, plasma bubbles that reached the ROCSAT- 1 altitude $(650 \mathrm{~km})$ usually ascended to altitudes $>848 \mathrm{~km}$. Also, the local times at which ROCSAT-1 saw the highest EPB rates coincided with those of the DMSP sun-synchronous orbits. Thus, the combined databases contribute a great deal to our understanding of the nonlinear growth and evolution of EPBs.

Conflicts seem to arise when we compare our DMSP climatology with others derived from ground-based radars and monitors of radiowave scintillations at fixed geographic locations. We believe these are attributable to the different, but related measured physical quantities. Plasma irregularities cover a wide range of scale sizes, from a few meters, seen by coherent backscatter radars, to a few hundred kilometers, observed as bubbles in the topside of the F-layer. Depending on wavelength and altitude, irregularities that cause scintillations range from a few hundred meters to a few kilometers. These and the meter-scale irregularities can appear in the topside of the F-layer where they are associated with EPBs, or they may be confined to the bottomside. Measurements from the JULIA radar in Peru show that bottomside irregularities are more common than the plumes that extend from them to altitudes far above the peak of the F-layer. Burke et al. (2003) compared the rates of EPBs detected by DMSP near the west coast of South America with the intensities of UHF scintillations observed at Ancon. They showed that when $\mathrm{S} 4>0.8$, EPB detections at $848 \mathrm{~km}$ and UHF scintillations followed the same pattern. In the C/NOFS era we hope to achieve sufficient understanding of this complex space weather phenomenon to predict its occurrence.

Acknowledgements. This work was supported under Air Force Office of Scientific Research task 2311SDA1, Air Force contract F19628-02-C-0012 with Boston College, and the National Polarorbiting Operational Environmental Satellite System Internal Government Studies Program.

Topical Editor M. Pinnock thanks V. Eccles and another referee for their help in evaluating this paper.

\section{References}

Aarons, J.: The longitudinal morphology of equatorial F-layer irregularities relevant to their occurrence, Space Sci. Rev., 63, 209-243, 1993.

Basu, B.: Generalized Rayleigh-Taylor instability in the presence of time-dependent equilibrium, J. Geophys. Res., 102, $17305-$ $17312,1997$.

Burke, W. J., Huang, C. Y., Valladares, C. E., Machuzak, J. S., Gentile, L. C., and Sultan, P. J.: Multipoint observations of equatorial plasma bubbles, J. Geophys. Res., 108, A5, 1221, doi:10.1029/2002JA009382, 2003.

Burke, W. J., Huang, C. Y., Gentile, L. C., and Bauer, L.: Seasonallongitudinal variability of equatorial plasma bubble occurrence, Ann. Geophys., 22, 3089-3098, 2004a,

SRef-ID: 1432-0576/ag/2004-22-3089.

Burke, W. J., Gentile, L. C., Huang, C. Y., Valladares, C. E., and $\mathrm{Su}, \mathrm{S}$. Y.: Longitudinal variability of equatorial plasma bubbles observed by DMSP and ROCSAT-1, J. Geophys. Res., 109, A12301, doi:10.1029/2004JA010583, 2004b.

Huang, C. Y., Burke, W. J., Machuzak, J. S., Gentile, L. C., and Sultan, P. J.: DMSP observations of equatorial plasma bubbles in the topside ionosphere near solar maximum, J. Geophys. Res., 106, 8131-8142, 2001.

Huang, C. Y., Burke, W. J., Machuzak, J. S., Gentile, L. C., and Sultan, P. J.: Equatorial plasma bubbles observed by DMSP satellites during a full solar cycle: Toward a global climatology, J. Geophys. Res., 107(A12), 1434, doi:1029/2002JA009452, 2002.

Luhmann, J. G. and Vampola, A. L.: Effects of localized sources on quiet time plasmasphere electron precipitation, J. Geophys. Res., 82, 2671-2676, 1977.

Rich, F. J. and Hairston, M.: Large-scale convection patterns observed by DMSP, J. Geophys. Res., 99, 3827-3844, 1994.

Scherliess, L. and Fejer, B. G.: Storm time dependence of equatorial disturbance dynamo zonal electric fields, J. Geophys. Res., 102, 24 037-24 046, 1997.

Sultan, P. J.: Linear theory and modeling of the Rayleigh-Taylor instability leading to the occurrence of equatorial spread F, J. Geophys. Res., 101, 26875-26891, 1996.

Tsunoda, R. T.: Control of the seasonal and longitudinal occurrence of equatorial scintillations by the longitudinal gradient in the integrated E-region Pedersen conductivity, J. Geophys. Res., 90, 447-456, 1985.

Zalesak, S. T. and Ossakow, S. L.: On the prospect for artificially inducing equatorial spread $F$, Memo. Rep. 4899, Nav. Res. Lab., Washington, D.C., Sept., 1982. 